\author{
И.Ю. Чернявский ${ }^{1}$, В.В. Тютюник ${ }^{2}$ В.Д. Калугин ${ }^{2}$, 3.В. Билык ${ }^{1}$, В.Б. Матыкин ${ }^{1}$ \\ ${ }^{1}$ Военный институт танковых войск НТУ «ХПИ», Харьков \\ ${ }^{2}$ Национальный университет гражданской защчитьл Украины, Харьков
}

\title{
ИСПОЛЬЗОВАНИЕ РЕЗУЛЬТАТОВ ПРИ РАЗРАБОТКЕ ТЕОРЕТИЧЕСКИХ И МЕТОДОЛОГИЧЕСКИХ ОСНОВ ПОСТРОЕНИЯ СИСТЕМЫ РАДИАЦИОННОГО МОНИТОРИНГА ЧРЕЗВЫЧАЙНЫХ СИТУАЦИЙ ВОЕННОГО ХАРАКТЕРА
}

В работе проведено выделение уровней иерархии информациинных связей, отдельных задач комплексной оценки очагов ядерного поражения и связывающих их информационных потоков. Анализируются причинно-следственные связи множества основных параметров, характер зависимости между уровнем ценности необходимой информации и ее количеством в системе радиационного мониторинга. Апробирована методология количественной оченки полного и точного учета необходимых параметров в задаче комплексной оченки очагов ядерного поражения. Авторами предлагается методология комплексной оченки очагов ядерного поражения на основе регистрации и учета необходимых и достаточных параметров ядерного взрыва, параметров среды и параметров объекта в зоне чрезвычайной ситуации.

Ключевые слова: теоретические и методологические основы построения, чрезвычайные ситуации военного характера, радиационный мониторинг, системно-информационный анализ, очаг ядерного поражения, параметры ядерного взрыва, выявление и оценка обстановки.

\section{Введение}

Постановка проблемы. В системе общегосударственных мероприятий по защите населения в условиях чрезвычайных ситуаций военного характера $[1,9]$ всё большую актуальность приобретает как заблаговременная подготовка к проведению спасательных и неотложных аварийно-восстановительных работ, так и достоверная оценка сложившейся в данной ситуации радиационной обстановки. К особенностям радиационного воздействия в результате атомных бомбардировок Хиросимы и Нагасаки относят: мгновенную реализацию основной части энергии ионизирующих излучений; наличие мощного нейтронного излучения и незнание пострадавшими особенностей поражения при ядерном взрыве. Эти особенности радиационного воздействия и определили характер радиологической защиты, осуществленной по принципу организации «косвенных контрмер», когда эти меры не могут изменить дозу облучения, но могут изменить качество жизни (состояние здоровья), и тем самым снизить вероятность реализации неблагоприятных эффектов облучения. Поэтому считается, что в условиях воздействия радиационного фактора военного характера [1-10] оперативное выявление и достоверная оценка ситуации являются взаимосвязанными этапами, определяющими эффективность принятия управленческого решения региональными штабами и службами гражданской защиты.

Оценка ситуации методом прогнозирования путем «идеализации» возможной радиационной обстановки [2] в тех или иных конкретных условиях может считаться вполне удовлетворительной, и годится лишь для решения задач общего планирова- ния, как правило, действий для военизированных формирований, основной задачей которых является выполнение поставленных аварийно-спасательных работ в очагах ядерного поражения (ОЯП).

Проведенный анализ эффективности применения существующих методик по оценке возможных чрезвычайных ситуаций военного характера $[2,9,10$, 25] показывает, что значительные ошибки при решении таких задач в современных условиях связаны:

- с применением методик и моделей, разработанных для оценки возникающих ситуаций, характерных для сценариев боевых действий с массированным применением ядерного оружия [31]. Существующие методики не учитывают тип ядерного боеприпаса, неспособны оценивать воздействие нестандартных боеприпасов, боеприпасов на новых физических принципах.

- с отсутствием комплексной многокритериальной оценки степени поражения населения при воздействии проникающей радиации (ПР) ядерного взрыва, зон радиоактивного заражения местности (РЗМ) и радиационно- опасных районов с наведенной активностью (НА). В специальной литературе под термином «радиационная обстановка» подразумевают оценку лишь радиационно-опасных участков местности, выявление которых позволяло бы своевременно и целенаправленно привести в действие комплекс мероприятий по защите населения от воздействия ионизирующих излучений на РЗМ (режимы радиационной защиты), и не учитываются дозовые нагрузки, получаемые населением в результате мгновенного облучения гамма-нейтронным излучением ПР. Методология оценки масштабов радиоактивно зараженной местности для разных видов ядерного взрыва, метеорологических условий достаточно хорошо проработана в специаль- 
ной литературе [1-9, 25]. Динамика и площади формирования радиационно-опасных районов с НА определяются соотношением реакций деления и синтеза данного типа ядерного боеприпаса, зависят от энергии нейтронной компоненты ПР, высоты взрыва, характера подстилающей поверхности (почвы). Указанные условия не отражены в современных методиках оценки радиационной обстановки.

- с отсутствием четко организованной системы прогнозирования доз ПР и радиационных потерь в очагах комбинированного ядерного поражения с выделением ведущего поражения: с преимущественно радиационно-механическим поражением (РМП); радиационно-термическим поражением (РТП); с комбинированным радиационным поражением (КРП); с радиационным поражением в «чистом» виде [27]. Отсутствие корректной оценки радиационного поражения в «чистом» виде связано, на наш взгляд, с отсутствием моделей прохождения гамма-нейтронного излучения ПР в пространстве и воздействия импульсного гамма-нейтронного излучения на человека, которые учитывают неравномерность облучения [26, 28, 30]. Проблемы оценки комбинированного поражения населения обычно связаны с отсутствием априорной информации о доминировании одного из поражающих факторов ядерного взрыва в интересующем для нас районе, что, в свою очередь, также связано с параметрами ядерного взрыва, типом ядерного боеприпаса.

- с отсутствием надежной и своевременной информации о параметрах ядерного взрыва, а также с отсутствием в настоящее время эффективных войсковых методов регистрации параметров взрывов малых и сверхмалых калибров, нейтронных боеприпасов [32]. Данное информационное звено является исходным источником достоверных данных для единой государственной системы выявления и оценки масштабов и последствий применения оружия массового поражения (ЕСВОП).

Надежность полученных данных существующих методик оценки уровня чрезвычайных ситуаций определяет порядок и особенности восстановительных и аварийно-спасательных работ, он также связан с особенностями ОЯП, что делает вопросы комплексной оценки ситуации в очагах ядерного поражения особо важными в современных условиях и требующими детальной проработки.

С учетом изложенных результатов анализа возможностей методик по выявлению и оценке обстановки в ОЯП для поддержки принятия решения целесообразно создание специализированной системы радиационного мониторинга параметров радиационного фактора, которая позволяет моделировать сложившуюся ситуацию, корректно оценивать масштабы комбинированного поражения и прогнозировать развитие последствий. Такой комплексный подход при оценке обстановки в ОЯП, с нашей точки зрения, возможен благодаря существующим зависимостям между закономерностями в развитии ядерного взрыва боеприпасов различного типа, мощности и вида взрыва [3-7, 10-18, 20-24] и параметрами импульсного гамма-нейтронного излучения проникающей радиации.

В научно-технической литературе существуют работы, посвященные разработке и созданию системы выявления и оценки радиационной обстановки для обеспечения безопасности жизнедеятельности населения [31, 33], в которых рассматриваются критерии для построения системы радиационного мониторинга, основанной на прогнозировании степени радиационного поражения населения по данным регистратора параметров радиационного фактора. Однако, они пока не раскрывают в достаточной мере вопросов информационного обеспечения, определяющих и обосновывающих структуру системы радиационного мониторинга.

Необходимо отметить, что управление сложными технологическими процессами, а, тем более, систем мониторинга, отвечающих за своевременное выявление и качественную оценку обстановки в условиях чрезвычайных ситуаций военного характера, до сих пор реализуется в условиях недостатка информации. Поэтому для достижения целей с помощью системы радиационного мониторинга весьма важно, чтобы в соответствующие центры обработки информации стекалась только необходимая (достаточная по объему) информация.

В данной работе предпринята попытка разработать теоретические и методологические основы построения таких систем (мониторинга) на основе системно-информационного анализа [34]. Поэтому проанализирован характер зависимости между уровнем ценности информации и ее количеством в системе радиационного мониторинга. Кроме того, в работе проведено выделение уровней иерархии информационных связей, отдельных задач комплексной оценки очагов ядерного поражения и связывающих их потоков информации. Изложена и апробируется методология количественной оценки полноты и точности учета необходимых параметров в задачах комплексной оценки очагов ядерного поражения.

\section{Изложение основного материала}

Методы оценки очагов ядерного поражения по времени проведения целесообразно подразделить на две группы: методы, основанные на анализе параметров ядерного взрыва с последующим моделированием радиационной ситуации (метод прогнозирования), и методы, основанные на выявлении фактической радиационной обстановки, т.е на апостериорных оценках (оценке последствий уже произошедшей чрезвычайной ситуации). Под апостериорной оценкой надо пониматьвесь комплекс мероприятий, проводимый силами специализированных формирований: ведение радиационной разведки местности, контроль доз облучения (дозиметрический контроль), контроль радиоактивного заражения различных предметов (населения), радиометрический анализ продуктов питания, воды и фуража $[1,2]$. На рис. 1 
приведена ориентировочная оценка изменения уровня радиации на местности в зависимости от времени, прошедшего после ядерного взрыва, а также представлены моменты времени начала формирования радиационно-опасных районов местности $\left(\mathrm{t}_{0}\right)$, начала $\left(\mathrm{t}_{\text {нразв}}\right)$ и конца $\left(\mathrm{t}_{\text {к.разв }}\right)$ фактической радиационной разведки местности силами специализированных формирований гражданской защиты.

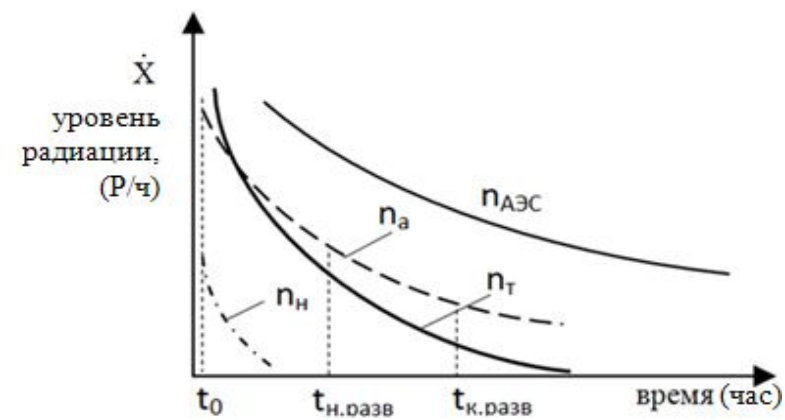

Рис. 1. Ориентировочная оценка изменения уровня радиации на местности в зависимости от времени, прошедшего после взрыва ядерного боеприпаса данного типа (показателя степени $\mathrm{n}$ ):

$\mathrm{n}_{\mathrm{H}} \approx 1,8-2,5$ - для взрыва нейтронного боеприпаса; $\mathrm{n}_{\mathrm{T}}=1,3$ - для термоядерного взрыва;

$\mathrm{n}_{\mathrm{a}}=1,2$ - для воздушного атомного взрыва;

$\mathrm{n}_{\text {Аэс }}=0,4$ для наземного атомного взрыва

и АЭС с реактором типа ВВЭР $[10,13,25]$

Уровни радиации для различных типов взрывов рассчитываются по уравнению:

$$
\dot{\mathrm{X}}_{\mathrm{t}}=\dot{\mathrm{X}}_{0}\left(\mathrm{t} / \mathrm{t}_{0}\right)^{-\mathrm{n}},
$$

где $\dot{\mathrm{X}}_{0}$ и $\dot{\mathrm{X}}_{\mathrm{t}}-$ уровни радиации (мощность экспозиционной дозы гамма-излучения) на моменты времени $\mathrm{t}_{0}$ и $\mathrm{t}$ соответственно.

На основе данных о процессе формирования радиационно-опасных районов местности и конкретного фактического времени получения данных о фактической радиационной обстановке оценку ОЯП на основе анализа параметров ядерного взрыва целесообразно считать не только комплексной, но и в достаточной степени «априорной» по отношению к результату выявления фактической радиационной обстановки.

С другой стороны, априорная оценка, как отмечалось ранее в работе [31], связана с тем, что имеется не только заранее подготовленная база данных о параметрах ядерных боеприпасов, среды распространения поражающих факторов ЯВ, подстилающей поверхности (почвы), о параметрах оцениваемого объекта (коэффициент защищенности и т.д.), но и просчитанные возможные ситуации $\left(\mathrm{S}_{\mathrm{i}}\right)$, которые могут сложиться в очагах ядерного поражения. Если число возможных ситуаций (сценариев комбинированного поражения населения) невелико, то именно для этих ситуаций можно заранее построить многомерное пространство состояний на основании параметров ядерного взрыва $\left(\mathrm{X}_{\mathrm{sв}}\right)$, параметров среды $\left(\mathrm{X}_{\mathrm{cpes}}\right)$, параметров объекта ( $\mathrm{Y}_{\text {объек }}$ и оперативно решить задачу выявления и оценки обстановки в ОЯП (рис. 2).

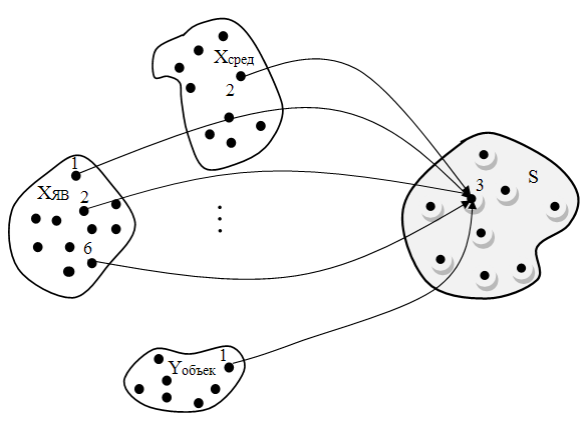

Рис. 2. Ситуационное управление при выявлении и оценке обстановки

Из вышеизложенного следует, что можно заготовить необходимый классификатор ситуаций в виде таблицы вероятных сценариев поражения населения, связанный с особенностями развития и доминированием поражающих факторов боеприпасов определенного типа, мощности и вида ЯВ. Данный факт позволяет оперативно выявлять и оценивать сложившуюся обстановку, применяя метод ситуационного управления [35]. На основе анализа работ, связанных с физикой ядерных взрывов, особенностями действия поражающих факторов ядерного взрыва [3-6, 14, 15, 21-24], а также на основе радиологических исследований [26, 28, 29] нами предложена медико-тактическая характеристика наиболее вероятных ситуаций (табл.1).

В табл. 1 возможные ситуации характеризуются параметрами ядерного взрыва (мощность и вид ядерного взрыва, тип ядерного боеприпаса, спектральные характеристики гамма-нейтронного излучения проникающей радиации, соотношение между гамманейтронным излучением); ожидаемыми санитарными потерями при данной форме острой лучевой болезни (с максимальной дозой гамма-нейтронного облучения); наличием радиационно-опасных районов местности (с показателем степени спада активности). В графе «спектральные характеристики» в скобках указаны проценты доминирующего спектра излучения. В графе «радиационное поражение» в скобках указан процент комбинированного поражения.

Учитывая вышеизложенное, система радиационного мониторинга чрезвычайных ситуаций военного характера, основанная на анализе параметров ядерного взрыва с последующим использованием классификатора ситуаций комплексной оценки ОЯП должна базироваться на технологиях, которые включают: технологии мониторинга (наблюдения) за состоянием среды, связанной с измерением параметров ядерного взрыва; технологии математического моделирования - оценки (обработки) измеренной информации на основе экспертно- аналитических моделей; геоинформационные технологии - создание банка данных и констант для работы математических моделей, интерпретация первичной информации, обработка данных для последующего использования в расчетах, моделировании и прогнозах. Как вариант, на рис. 3. представлена предлагаемая нами методология комплексной оценки очагов ядерного поражения. 
Медико-тактическая характеристика ситуаций при применении ядерного оружия

\begin{tabular}{|c|c|c|c|c|c|c|}
\hline \multirow{2}{*}{\multicolumn{2}{|c|}{ Характеристика параметров }} & \multicolumn{5}{|c|}{ Ситуации } \\
\hline & & $\mathrm{S}_{1}$ & $\mathrm{~S}_{2}$ & $\mathrm{~S}_{3}$ & $\mathrm{~S}_{4}$ & $\mathrm{~S}_{5}$ \\
\hline \multicolumn{2}{|c|}{ мощность ЯВ, кт } & $0,1-1$ & $0,1-5$ & $5-20$ & $50-500$ & $500-1000$ \\
\hline \multicolumn{2}{|c|}{ вид ЯВ } & $\mathrm{B}$ & $\mathrm{B}$ & $\mathrm{B}$ & $\mathrm{H}, \Pi$ & $\mathrm{H}, \Pi$ \\
\hline \multirow{2}{*}{$\begin{array}{l}\text { спектральные } \\
\text { характеристики }\end{array}$} & $\begin{array}{l}\text { энергия нейтро- } \\
\text { нов } E_{n}, \text { МэB } \\
\end{array}$ & $\begin{array}{l}2,3-1,1 \\
(15,4 \%)\end{array}$ & $14-12,2(40,5 \%)$ & $\begin{array}{c}2,3-1,1 \\
(15,4 \%)\end{array}$ & $\begin{array}{l}2,3-1,1 \\
(9,03 \%)\end{array}$ & $\begin{array}{l}2,3-1,1 \\
(9,03 \%)\end{array}$ \\
\hline & $\begin{array}{c}\text { энергия нейтро- } \\
\text { нов } \mathrm{E}_{\gamma}, \text { МэВ } \\
\end{array}$ & $2-1(43 \%)$ & $2-1(33,2 \%)$ & $2-1(43 \%)$ & $2-1(43 \%)$ & $2-1(43 \%)$ \\
\hline \multicolumn{2}{|c|}{ тип ЯБ } & атомный & нейтронный & атомный & термоядерный & термоядерный \\
\hline \multicolumn{2}{|c|}{ ОЯП } & КРП & КРП & РТП & PTM & термич.пораж. \\
\hline \multirow{2}{*}{$\begin{array}{l}\text { примерный вклад } \\
\text { в общую дозу \% }\end{array}$} & $\mathrm{n}$ & 70 & 90 & 70 & 40 & 30 \\
\hline & $\gamma$ & 30 & 10 & 40 & 60 & 70 \\
\hline \multirow{2}{*}{$\begin{array}{l}\text { санитарные } \\
\text { потери в \% }\end{array}$} & $\begin{array}{l}\text { радиационные } \\
\text { поражение }\end{array}$ & $\begin{array}{r}95-97 \\
(5-3)\end{array}$ & $\begin{array}{c}100 \\
\text { (не ожид.) }\end{array}$ & $\begin{array}{c}40-70 \\
(60-30) \\
\end{array}$ & $\begin{array}{l}\text { одиночные } \\
(50-30)\end{array}$ & $\begin{array}{l}\text { не ожид. } \\
(5-3)\end{array}$ \\
\hline & $\begin{array}{c}\text { термические } \\
\text { ожоги }\end{array}$ & не ожидается & $\begin{array}{c}\text { не } \\
\text { ожидается }\end{array}$ & одиночные & $50-70$ & $95-97$ \\
\hline \multicolumn{2}{|c|}{$\begin{array}{c}\text { максимальная доза в районе } \\
\text { санитарных потерь, Гр }\end{array}$} & $\begin{array}{c}50 \\
\text { и более } \\
\end{array}$ & $\begin{array}{c}60-120 \\
\text { и более } \\
\end{array}$ & 15 & 6 & 6 \\
\hline \multicolumn{2}{|c|}{ формы ОЛБ } & $\begin{array}{c}\text { церебральная } \\
\text { кишечная } \\
\text { сосудистая } \\
\end{array}$ & $\begin{array}{c}\text { ранняя преходя- } \\
\text { щая недееспо- } \\
\text { собность (РПН) } \\
\end{array}$ & $\begin{array}{l}\text { кишечная } \\
\text { сосудистая }\end{array}$ & $\begin{array}{c}\text { кишечная } \\
\text { костно-мозговая }\end{array}$ & $\begin{array}{c}\text { кишечная } \\
\text { костно-мозговая }\end{array}$ \\
\hline \multicolumn{2}{|c|}{$\begin{array}{c}\text { радиационно-опасные районы ме- } \\
\text { стности }\end{array}$} & HA, P3M & HA & HA, P3M & HA, P3M & P3M \\
\hline \multicolumn{2}{|c|}{$\begin{array}{c}\text { показатель степени спада активно- } \\
\text { сти, } \mathrm{n}\end{array}$} & 1,2 & $1,8-2,5$ & 1,2 & 1,3 & 1,3 \\
\hline
\end{tabular}

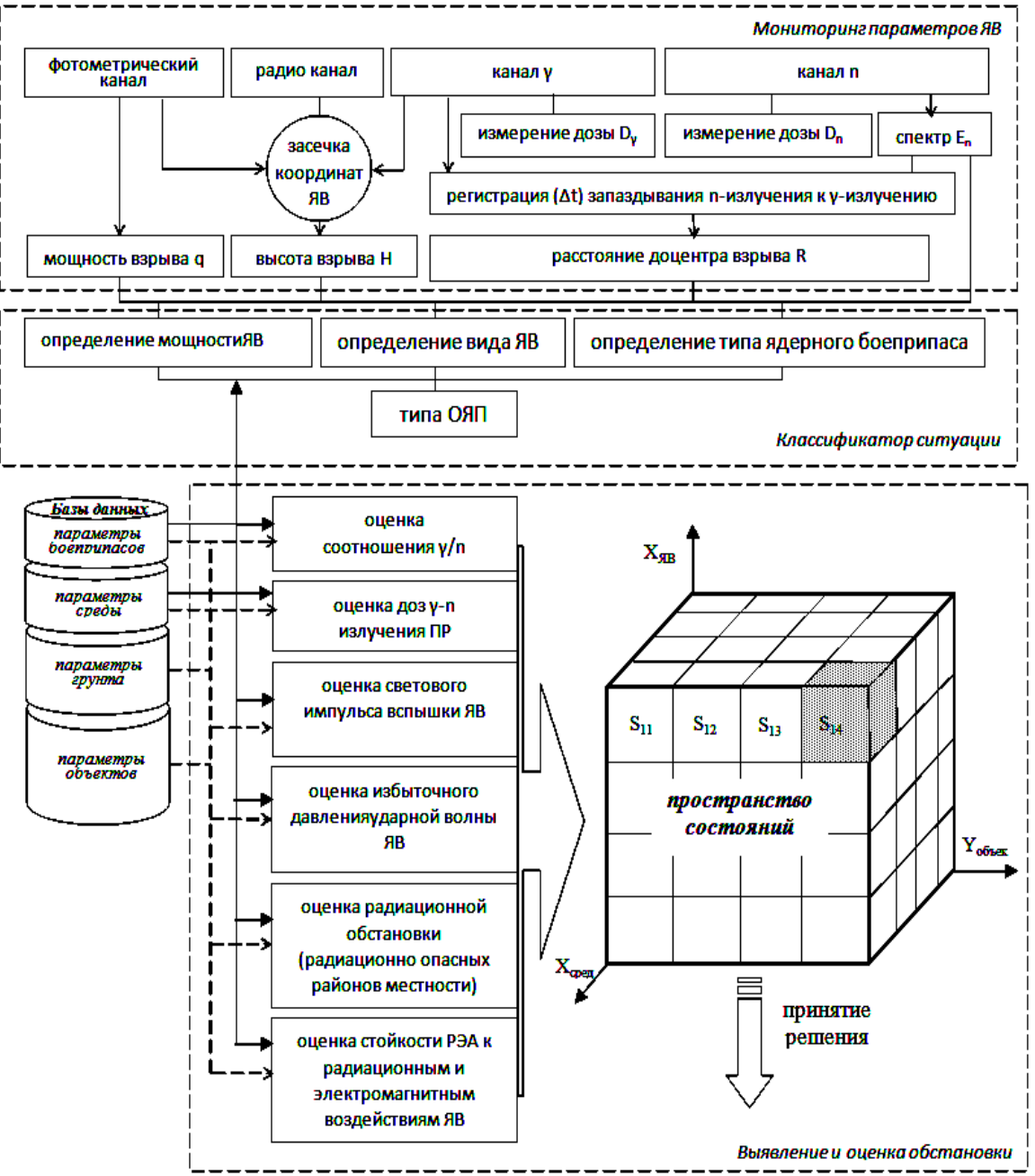

Рис. 3. Методология комплексной оценки очагов ядерного поражения в системе радиационного мониторинга
Функционально необходимо выделить модуль мониторинга параметров ЯВ (фотометрический канал, радиоканал, канал гамма-нейтронного излучения ПР) [31, 32], модуль классификатора ситуации и модуль выявления и оценки сложившейся обстановки. Отдельным функциональным элементом служит специализированная база данных параметров ядерных боеприпасов (атомный, термоядерный, нейтронный), параметров среды (атмосферы), параметров грунта, параметров объекта.

Комплексная оценка включает:

- оценку биологического воздействия гамма-нейтронного излучения ПР ядерного взрыва всех типов боеприпасов с учетом неравномерности облучения на заданном расстоянии от центра взрыва и энергетических спектров излучений;

- оценку границ зон поражения разной степени тяжести световым излучением ЯВ; 
- оценку границ зон поражения разной степени тяжести избыточным давлением ударной волны ЯВ;

- выявление и прогнозирование границ радиационно-опасных участков местности (зон и районов), формирующихся как в результате загрязнения продуктами радиоактивного распада, так и районов с НА, образующихся в результате воздействия нейтронной компоненты ПР; оперативное определение показателя степени спада (n) активности в указанных районах (рис.1); расчет уровней радиации на заданный период времени (выражение (1) с последующим прогнозированием дозовой нагрузки;

- оценку стойкости радиоэлектронной аппаратуры (РЭА) инфраструктуры в ОЯП к радиационным и электромагнитным воздействиям ЯВ.

В общем случае комплексная оценка ОЯП представляет собой исследовательский и расчетноаналитический процесс, целью которого является получение данных о потерях населения в виде приведенных зон гамма-нейтронного облучения, а также о характере развития прогнозируемого явления (наличие радиационно-опасных районов) и определяющих параметрах функционирования систем или объектов инфраструктуры. Использование предложенной методологии радиационного мониторинга (рис. 3), основанной на анализе параметров ядерного взрыва с последующим использованием классификатора ситуаций, в состоянии обеспечить необходимый уровень оперативности выявления и оценки ситуации при наличии только оптимального объема и качества информации.

Для анализа большинства практических задач комплексной оценки в системе радиационного мониторинга достаточным набором информационных показателей будут количество единиц (элементов) информации (I), качество (ценность) единицы информации с точки зрения достижения определенных целей $(\lambda)$ и себестоимость единицы информации. Производными от этого минимального набора показателей являются, согласно работам Ю.М. Горского [34], количество взвешенной по полезности (ценности) информации (I') и затраты на получение единицы информации. В общем случае I' будет некоторой функцией (в частном случае I'= $\mathrm{f}(\mathrm{I} \lambda)$ ). На рис.4 представлен характер зависимости между ценностью получаемой информации при комплексной оценке очагов ядерного поражения и её количеством. Важным аспектом характера информации является нелинейность полезной информации, т.е уменьшение ценности информации $\left(\lambda_{1}=\varphi(\mathrm{I})\right)$ с возрастанием ее количества. Падение «удельной» ценности информации связано с перенасыщением ее осведомляющей информацией, которая не всегда является необходимой для удовлетворительного функционирования системы радиационного мониторинга. В отдельных случаях, когда I' совпадает с I, тогда $\lambda_{2}=\mathrm{const}=1$, - это является необходимым и достаточным объемом информации для корректного выявления и оценки сложившейся ситуации в ОЯП.
Область, находящуюся под кривой информативности I' (рис. 4), будем определять как область четких данных о параметрах ядерного взрыва $\left(\mathrm{X}_{\text {яв }}\right)$, параметрах среды $\left(\mathrm{X}_{\text {сред }}\right)$, параметрах объекта $\left(\mathrm{Y}_{\text {объек }}\right)$ т.е область данных, характерных только для конкретной ситуации. Соответственно, область над кривой I' - это область нечетких данных, в ней могут находиться данные других ситуаций.

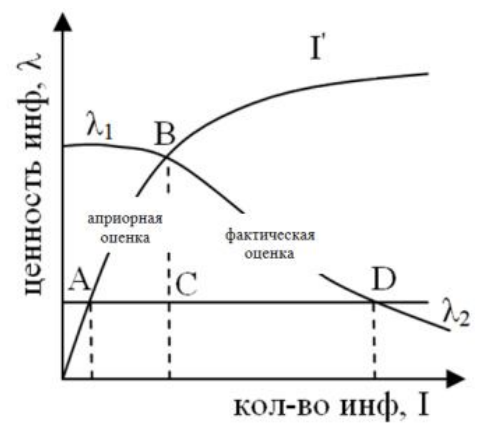

Рис. 4. Характер зависимости между ценностью информации и ее количеством

Область, в которой происходит накопление и, соответственно, увеличение объема информации при радиационном мониторинге, целесообразно определить как область необходимой и достаточной информации. Для точки А (рис.4) данный объем достаточен для выявления и оценки сложившейся ситуации, а, следовательно, и для принятия первого обоснованного решения. Увеличение количества информации дает возможность достичь области оптимального объема и качества информации для взвешенного выявления и оценки сложившейся ситуации в границах заданного времени (область $\mathrm{ABC}$ ). Точка В соответствует оптимальному отношению количества полученной информации с еe ценностью - область оптимальной информации.

Область (BCD), в которой увеличение количества информации приводит к уменьшению ее качества, характеризуется уменьшением ценности информации:

- за счет увеличения времени на обработку информации в системе радиационного мониторинга, а также на оценку ее ценности;

- за счет нахождения информации в области нечетких данных с последующей классификацией ее как излишней;

- за счет увеличения времени на выявление и оценку сложившейся ситуации.

Кроме того, проведенный анализ показывает, что при выявлении и оценке обстановки информация со временем теряет свою ценность за счет обесценивания информации (например, из-за несвоевременного проведения радиационной разведки местности либо из-за превышения допустимых временных параметров на ее проведение), старения информации из-за задержек при ее передаче или переработке.

Точка D является критической точкой, когда количество полученной информации является предельно-допустимой для надежного и своевременного вы- 
явления и оценки ситуации, далее начинается область лишней, «ненужной» информации. Исходя из вышеизложенного, предлагается использовать следующую модель оценки уровня информативности необходимой в системе радиационного мониторинга информации:

$$
\mathrm{I}=\mathrm{f}(\lambda, \mathrm{t}, X, \mathrm{~T}),
$$

где $\lambda$ - ценность (полезность) поступающей информации; $\mathrm{t}$ - затраченное время на получение информации; $\mathrm{X}$ - характеристика информации: четкие или нечеткие данные; T - период, за который может быть выявлена и оценена ситуация при наличии таких данных.

Систему радиационного мониторинга невозможно представить одной моделью - необходима целая иерархия моделей, которые будут различаться по уровню отображаемых явлений. Поэтому проанализируем необходимую базу знаний с целью оптимизации информационного состава объема, структуры и свойств системы радиационного мониторинга чрезвычайных ситуаций военного характера. По нашим оценкам, такой процесс позволит уже на ранних этапах исследования «отсечь» заведомо неприемлемые концепции комплексной оценки ОЯП, выделить «узкие места», подлежащие дальнейшей проработке, устранить возникающие противоречия. На основе анализа полуэмпирических зависимостей, полученных при ядерных испытаниях и изложенных в открытой литературе [3-8, 10-24], а также на основании проведённых нами исследований по влиянию соотношений гамма-нейтронного излучения на корректную оценку действия ПР, нами построена иерархия задач (рис. 5), связанная с учетом основных параметров: ядерного взрыва, среды распространения и объекта, необходимых, с нашей точки зрения, для комплексной оценки очагов ядерного поражения при применении боеприпасов разного типа, мощности и вида ЯВ.

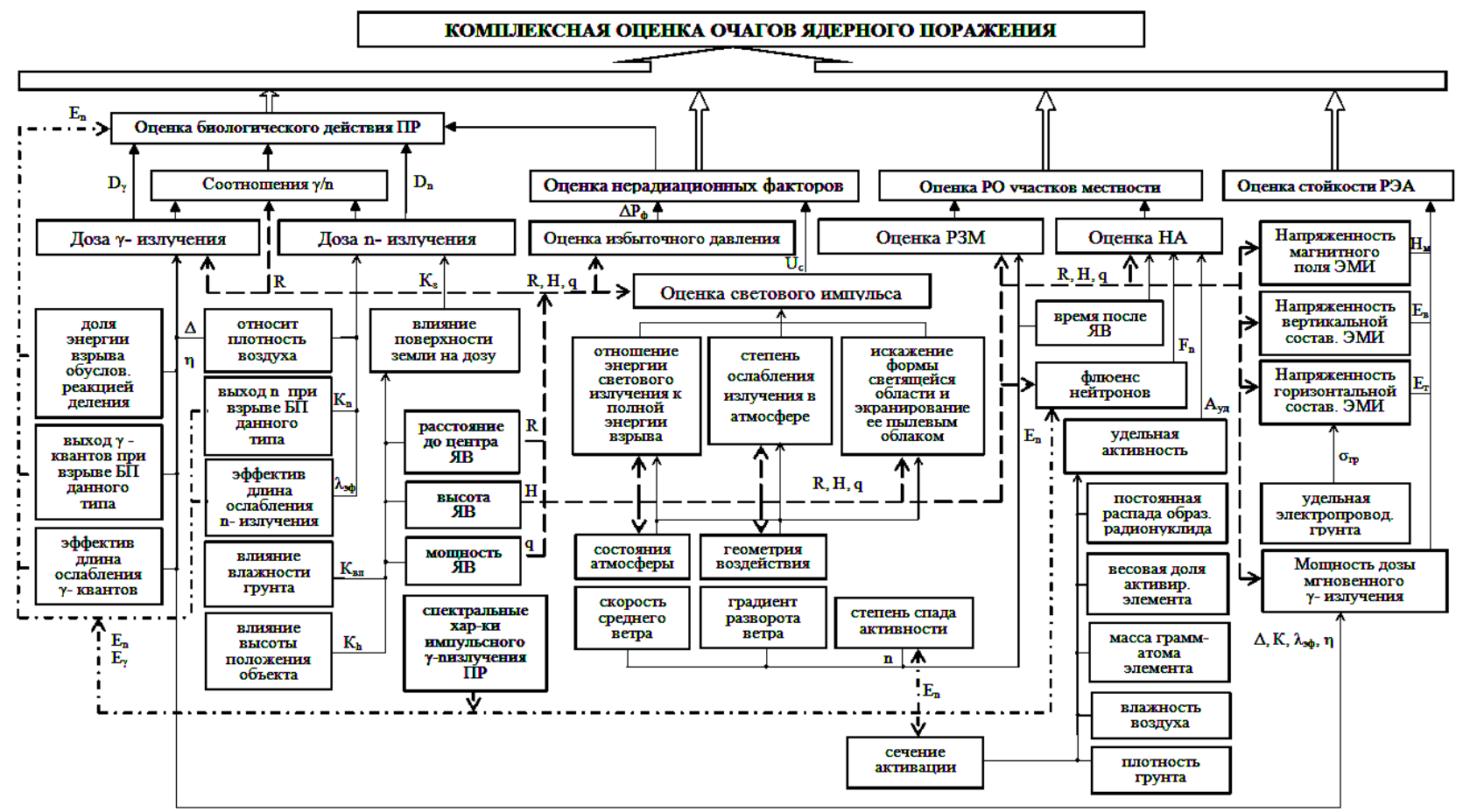

Рис. 5. Декомпозиция задачи учёта необходимых и достаточных параметров для комплексной оценки очагов ядерного поражения

Анализ проведенной декомпозиции показывает, что функционирование системы характеризуется причинно-следственными связями между множеством основных параметров. Порядка 90 \% всех учтенных параметров как при оценке биологического действия ПР на население (с учетом поражения от нерадиационных факторов ЯВ), так и при оценке возможных потерь на радиоционно-опасных (PO) участках местности, зависят от базовых параметров ядерного взрыва $X_{я в}$, расстояния до центра взрыва и высоты взрыва (т.е координаты ЯВ), мощности ЯВ. Выявлено, что определение такого параметра, как высота ЯВ, обеспечивает при оценках нерадиационных факторов учет не только метеорологических характеристик атмосферы (состояние атмосферы), но и вида ядерного взрыва.
Анализ перечисленных параметров ядерного взрыва показал, что несмотря на то, (как принято считать), что радиус зоны поражения ударной волной нейтронного боеприпаса меньше, чем при взрыве атомного боеприпаса той же мощности, проведенный нами анализ не выявил особенностей в оценке нерадиационных факторов ЯВ боеприпасов различного типа (атомный, термоядерный или нейтронный).

Однако, такой параметр, как спектральные характеристики ПР, необходим для определения как специфики прохождения гамма-нейтронного излучения в пространстве $\mathrm{X}_{\text {сред }}$ (параметры среды), так и специфики поражающего действия в очагах ядерного поражения, что отражается при учете типа ядерного боеприпаса (БП). Поэтому спектральные характеристики $\left(\mathrm{E}_{\mathrm{n}}\right)$ нейтронного излучения проникающей 
радиации является определяющими при выборе следующих параметров (рис. 5): выхода нейтронов при ЯВ данного типа; длины свободного пробега нейтронов ЯВ данного типа; флюенса нейтронов; сечения активации для элементов грунта; степени спада активности на зараженной местности. Для количественной оценки полноты и точности учета необходимых параметров в задачах по комплексной оценке ОЯП, представленных на рис. 5, будем использовать показатель, вычисляемый по выражению [36]:

$$
\mathrm{K}_{\text {полн }}=1-\sum_{\mathrm{j}=1}^{4} \xi_{\mathrm{j}} \sum_{\mathrm{i} \in \gamma_{\mathrm{i}}} \lambda_{\mathrm{i}},
$$

где $0<\lambda_{\mathrm{i}}<1-$ вес (ценность) і-го параметра; $\gamma_{\mathrm{i}}-$ множество параметров, которые учитываются ј-м способом обобщения; $\xi_{\mathrm{j}}-$ погрешность, обусловленная неточным (обобщением) учётом параметра. Значение относительной погрешности $\xi_{\mathrm{j}}(\mathrm{j}=1,2,3$, 4) зависит от способа учёта параметров и обычно находится в пределах:

$$
\xi_{\mathrm{j}}=\left\{\begin{array}{l}
0-\text { при непосредственном }(\mathrm{H}) \text { учете }(\mathrm{j}=1) ; \\
0,4-\text { при простом }(\text { П) обобщении }(\mathrm{j}=2) ; \\
0,6-\text { при функциональном }(\Phi) \text { обобщении }(\mathrm{j}=3) ; \\
1,0-\text { при косвенном }(\text { К) обобщении }(\mathrm{j}=4) .
\end{array}\right.
$$

Непосредственным способом учёта параметра является измерение спектральных характеристик гамма- нейтронной компоненты, т.е. определение энергии воздействующего излучения прямым спектрометрическим методом $\left(\mathrm{E}_{\gamma / \mathrm{n}}\right)$.

Простое обобщение связано с присвоением некоторого значения из заранее прописанных величин, взятых из баз данных. Например, для зарегистрированных значений энергии нейтронного излучения таким параметром будет доля энергии взрыва, обусловленная реакцией деления (ๆ) или эффективная длина ослабления нейтронного излучения $\left(\lambda_{\text {эф}}\right)$ (рис. 5).

Функциональное обобщение заключается в аналитическом вычислении необходимого параметра с учётом других зависимостей и всех возможных поправочных коэффициентов. К такого рода обобщению целесообразно отнести определение поглощенной дозы гамма-излучения $\left(\mathrm{D}_{\gamma}\right)$ или флюенса нейтронов $\left(\mathrm{F}_{\mathrm{n}}\right)$ на заданном расстоянии от центра взрыва, влияния поверхности Земли на определение значения поглощенной дозы нейтронного излучения $\left(\mathrm{K}_{3}\right)$.

При косвенном учёте (обобщении) оценка параметра формируется в условиях неопределённости информации об объекте, в разряд которой попадает положение тела человека по отношению к источнику (здесь для получения граничной оценки наиболее пессимистического прогноза можно принять наихудшую ситуацию). Данный подход к оценке полноты и точности учета необходимых параметров позволяет ранжировать их как по способу получения, так и по важности (ценности) их учета в предлагаемых моделях.

Учитывая информационно-логические связи в представленной на рис. 5 иерархии, нами получено ранжирование основных параметров, влияющих на корректную оценку биологического действия гамманейтронного излучения ПР, и способы их учёта в системе радиационного мониторинга (табл.2). При ранжировании были выделены только прямые связи, влияющие на оценку биологического действия гамма- нейтронного излучения ПР. Такой параметр, как поглощенная доза гамма-нейтронного излучения, не представлен, однако учтены параметры и коэффициенты, его определяющие, по известным в литературе выражениям [3-8,10-24].

Таблица 2

Ранжирование основных параметров, влияющих на корректную оценку биологического действия гамма- нейтронного излучения ПР, и способ их учёта в системе радиационного мониторинга

\begin{tabular}{|c|l|c|c|}
\hline № & \multicolumn{1}{|c|}{ Наименование параметра } & Способ учёта & Вес параметра в относ. единицах \\
\hline 1 & Мощность ядерного взрыва (тротиловый эквивалент) & $\Phi$ & 0,25 \\
\hline 2 & Спектры гамма-нейтронного излучения & $\mathrm{H}$ & 0,2 \\
\hline 3 & Расстояние между человеком и центром ЯВ & $\mathrm{K}$ & 0,1 \\
\hline 4 & Высота ядерного взрыва & $\mathrm{H}$ & 0,1 \\
\hline 5 & Выход нейтронов при взрыве боеприпаса данного типа & $\Pi$ & 0,06 \\
\hline 6 & Соотношение компонентов гамма-нейтронного излучения $(\gamma / \mathrm{n})$ & $\mathrm{H}$ & 0,05 \\
\hline 7 & Выход $\gamma$ - квантов при взрыве боеприпаса данного типа & $\Pi$ & 0,05 \\
\hline 8 & Эффективная длина ослабления $\gamma$ - квантов & $\Pi$ & 0,05 \\
\hline 9 & Эффективная длина ослабления п- излучения & $\Pi$ & 0,05 \\
\hline 10 & Доля энергии взрыва, обусловленная реакцией деления & $\Pi$ & 0,04 \\
\hline 11 & Относительная плотность воздуха & $\Pi$ & 0,015 \\
\hline 12 & Влияние поверхности Земли на дозу & $\Phi$ & 0,015 \\
\hline 13 & Избыточное давление во фронте ударной волны & $\Phi$ & 0,01 \\
\hline 14 & Световой импульс вспышки ядерного взрыва & $\Phi$ & 0,01 \\
\hline
\end{tabular}

Примечания: Н - непосредственный учёт фактора (измерение); $\Phi$ - учёт фактора путём функционального обобщения (вычисление); П - простое обобщение; К -косвенное обобщение

Используя выражение (3) и полученные весовые значения (табл. 2), оценим полноту и точность учёта различных параметров в предложенной иерархии: 


$$
\begin{gathered}
\quad \kappa_{\text {полн }}=1-\left(\lambda_{1}(0,25) \cdot \xi_{3}(0,6)+\lambda_{2}(0,2) \cdot \xi_{1}(0)+\lambda_{3}(0,1) \cdot \xi_{4}(1)+\lambda_{4}(0,1) \cdot \xi_{1}(0)+\lambda_{5}(0,06) \cdot \xi_{2}(0,4)+\right. \\
+\lambda_{6}(0,05) \cdot \xi_{1}(0)+\lambda_{7}(0,05) \cdot \xi_{2}(0,4)+\lambda_{8}(0,05) \cdot \xi_{2}(0,4)+\lambda_{9}(0,05) \cdot \xi_{2}(0,4)+\lambda_{10}(0,04) \cdot \xi_{2}(0,4)+ \\
+\lambda_{11}(0,015) \cdot \xi_{2}(0,4)+\lambda_{12}(0,015) \cdot \xi_{3}(0,6)+\lambda_{13}(0,01) \cdot \xi_{3}(0,6)+\lambda_{14}(0,01) \cdot \xi_{3}(0,6)=1-0,377=0,623 .
\end{gathered}
$$

Аналогичным образом определяется полнота и точность учёта представленных в иерархии параметров, необходимых для оценки нерадиационных факторов, радиационно-опасных районов местности и стойкости радиоэлектронной аппаратуры.

Таким образом, апробирована методология количественной оценки полного и точного учета необходимых параметров в задаче комплексной оценки очагов ядерного поражения.

\section{Выводы}

1. Показано, что система радиационного мониторинга чрезвычайных ситуаций военного характера базируется на общих принципах технологии мониторинга за состоянием среды; использования технологии математического моделирования - оценки (обработки) измеренной информации на основе экспертноаналитических моделей; на результатах геоинформационных технологий (создание банка данных и констант для работы математических моделей, интерпретация первичной информации, обработка данных для последующего использования в расчетах, моделировании и прогнозах).

2. Установлено, что использование априорного метода при обработке результатов моделирования, основанного на анализе параметров ядерного взрыва с последующим использованием классификатора ситуаций, в состоянии обеспечить необходимый уровень оперативности выявления и оценки ситуации при наличии оптимального объема и качества информации. На основе анализа характера зависимости между ценностью информации и ее количеством предложена модель оценки информативности, необходимой в системе радиационного мониторинга.

3. Проведена количественная оценка полноты и точности учета необходимых параметров в задачах по оценке биологического действия гамма- нейтронного излучения проникающей радиации на основании критерия, учитывающего способ учёта параметров, а также ценность данной информации в системе радиационного мониторинга.

\section{Список литературы}

1. Зашита объектов народного хозяйства от оружия массового поражения: Справочник / Под редакиией Г.П. Демиденко. 2-е изд., перераб. и доп. - К.: Вищашк. Головное изд-во, 1989.- 287 c.

2. Защита от оружия массового поражения / Под.ред. В.В. Мясникова. - 2-е изд., перераб. и доп. - М. : Воениздат. 1989. - 389 c.

под редакиией С.С. Григоряна и Г. С. Шапиро. - М.: Мир, $1971.680 \mathrm{c}$.
4. Действие ядерного оружия: Пер. с англ. - М.: Воениздат, 1965. - 683 с.

5. Иванов А.И., Рыбкин Г.И. Поражающее действие ядерного взрыва.- М.: Военное издательство МО Союза ССР, 1960. -378 c.

6. Иванов А.И., Науменко И.А., Павлов М.П. Ракетно-ядерное оружие и его поражающее действие. М., Воениздат, 1971. 224c.

7. Зыков И.К. Радиоактивные излучения ядерного взрыва.- изд. ЛВИКА им.А.Ф. Можайского. $-1970 .-108$ с.

8. Механическое действие ядерного взрыва. - М.: ФИЗМАТЛИТ, 2003.-384 с.

9. Гражданская оборона: Учебник для вузов/В. Г. Атаманюк, Л. Г. Ширшев, Н. И. Акимов. Под ред. Д. И. Михайлика. - М.: Высши. шк., 1986. -207 с:

10. Тыльков С.Д., Волков А.В. Оиенка обстановки в чрезвычайных ситуациях. Методические указания.-М. МИИТ, 2008.-32 c.

11. Оиенка устойчивости работы электронных систем при воздействии ионизирующих излучений: метод. пособие для практич. занятий по диси. «Защита населения и хозяйственных объектов в чрезвычайных ситуачиях. Радиационная безопасность»/А.Н. Навоша $и$ др. - Минск: БГУИР, 2008. -18 c.

12. Особенности проектирования электронных средств специального назначения [Электронный ресурс]: научно-образоват. модуль/Минобрнауки России, Самар. Гос.аэрокосм. ун-т им.С.П.Королева (нац. исслед. ун-т); авт.-сост. Г.Ф. Краснощекова. Электрон.текстовые и граф. дан.(3,87 Мбайт)- Самара, 2012.- 1 эл. опт. диск (CD-ROM).

13. Справочник по поражающему действию ядерного оружия. Часть 1. Поражающеее действие ядерных взрывов и оценка эффективности ядерных ударов.-М. Военное издательство.-1986. -608 c.

14. Боевые свойства ядерного оружия -М.: Военное издательство. $-1967 .-624$ c.

15. Днепровский А.П. Оружие массового поражения и защита авиационных подразделений. - изд. ВВИА им. Н.Е. Жуковского. $-1983 .-358$ c.

16. Лазарев Г.Н. Физико-технические принципь устройства и поражающее действие ядерного оружия. изд. КВВАИУ.-1982. -249 с

17. Волощенко А.М., Гончаров А.Н., Кондушкин Н.А. Влияние влажсности воздуха на характеристики ионизирующих излучений// Сб. научных трудов МИФИ: Защчта от ионизируюших излучений / Под ред. В.К. Сахорова. М.: Энергоатомиздат, 1988. С. 57-60.

18. Гаранюшкин Н.В., Кондурушкин Н.А. Банк данных для расчёта полей нейтронов и гамма-излучения от точечных и распределённых источников у гранищы раздела воздух-земля// Шестая Российская научная конференция по защчте от ионизирующих излучений ядерно-технических установок. Тезисы докладов. Обнинск: ГНЦ ФЭИ, 1994. Т.1. С. 93-94.

19. Распространение ионизирующих излучений в воздухе/ Климанов В.А., Коновалов С.А., Кочанов В.А. и др. Под ред. В.И. Кухатевича и В.П. Машковича.- М.: Атомиздат, 1979. - 216 c.

20. Кухтевич В.И. и др. Защчита от проникающей радиачии ядерного взрыва. - М. Атомизда, 1970-192 c.

21. Тарасенко Ю. Н. Ионизачионные методы дозиметрии высокоинтенсивного ионизирующего излучения M.: Техносфера, 2013. -264 c. 
22. Тарасенко Ю.Н. Вторичные эталоны единич измерения ионизирующих излучений. -M.: Техносфера, 2011. $-448 c$

23. Физика ядерного взрыва: Том 1. Развитие взрыва/ Министерство обороны Российской Федерации. Центральный физико-технический институт. -М.: Наука. Физматлит, 1997. -528 c.

24. Физика ядерного взрыва: Том 2. Действие взрыва/ Министерство оборонь Российской Федерации. Центральный физико-технический институт. -М.: Наука. Физматлит, 1997. -256 с.

25. STANAG 2103. Reporting nuclear detonations, biological and chemical attacks, and predicting and warning of associated hazards and hazard areas. ATP-45(A,C), NATO Volume 1-2,2005.

26. Дозовая нагрузка на человека в полях гамманейтронного излучения. М., Атомиздат, 1978.- 168 c. Авт.: Гозенбук В.Л., Кеирим-Маркус И.Б., Савинский А.К., Чернов E.H.

27. Хоруженко А.Ф. Комбинированные радиационные поражения при чрезвычайных ситуачиях мирного и военного времени. Стратегия гражданской защиты. проблемы и исследования. Выпуск №1. Том 4., 2014 г. С. 310-323.

28. Куиенко С.А., Бутомо Н.В., Гребенюк А.Н. и др. Военная токсикология, радиобиология и медииинская защита: Учебник/Под ред. С.А. Кученко. - СПб: ООО «Издательство ФОЛИАНТ», 2004. -528 c.

29. Клинические рекомендации по оказанию медицинской помощи пострадавшим от воздействия ионизирующего излучения в чрезвычайных ситуащиях. - М.: МЗ PФ.-2013. -72 c.

30. Радиационная медицин: учеб.пособие/А.Н. Гребеннюк, В.И. Легеза, В.И. Евдокимов, В.В. Салухов, А.А. Тимочевский; под. ред. С.С. Алексанина, А.Н. Гребенюка; Всерос. иентр. экстрен. и радиаи. Медиџины им. А.М. Никифорова МЧС России. -СПб.: Политехника-
сервис,2013.-Ч.2: Клиниеа, профилактика и лечение радиационных поражений.-156 c.

31. Чернявский И.Ю. Оченка степени радиачионного поражения путём прогнозирования дозовых нагрузок за данными датчика боеспособности / И.Ю. Чернявский, B.B. Марущченко, А.В. Матыкин// Системи озброєння $i$ військова техніка: науково - технічний журнал. - $X$.: Харківський університет Повітряних Сил імені Івана Кожедуба, 2016. - №1(45). - С. 125 - 133.

32. Чернявский И.Ю., Григорьев А.Н., Билькк 3.В., Матыкин В.Б. Применение кремниевых ріп детекторов для регистрации параметров ядерного взрыва. Системи озброєння $i$ військова техніка: науковий журнал. - X. Харківський університет Повітряних Сил імені Івана Кожедуба, 2016. - №4(48). - С. 61-68.

33. Чернявский И.Ю., Тютюник В.В., Калугин В.Д. Анализ условий для создания системы выявления и оценки уровня радиационной безопасности жизнедеятельности населения при чрезвычайных ситуациях военного характера. Збірка наукових прачь. Проблеми надзвичайних ситуацій. - Харків : НУЦЗУ, 2016. - Вип. 23. C. $168-185$.

34. Горский Ю.М. Системно-информационный анализ процессов управления / Ю.М. Горский. - М., 1988. $322 c$.

35. Поспелов Д.А. Ситуачионное управление: теория и практика. - М.: Наука.-Гл. ред. Физ.-мат. Лит., 1986. $288 \mathrm{c}$.

36. Городнов В.П. Моделирование боевых действий частей, соединений и объединений войск ПВО / В.П. Городнов. - Х.: ВИРТА ПВО, 1987. -380 c.

Надійшла до редколегї 27.12.2017

Рецензент: д-р техн. наук, проф. О.В. Стаховський, Військовий інститут танкових військ Національного технічного університету «ХПІ», Харків.

\section{ВИКОРИСТАННЯ РЕЗУЛЬТАТІВ ПІД ЧАС РОЗРОБКИ ТЕОРЕТИЧНИХ ТА МЕТОДОЛОГІЧНИХ ОСНОВ ПОБУДОВИ СИСТЕМИ РАДІАЦІЙНОГО МОНІТОРИНГУ НАДЗВИЧАЙНИХ СИТУАЦІЙ ВОЄННОГО ХАРАКТЕРУ}

І.Ю. Чернявський, В.В. Тютюник, В.Д. Калугін, З.В. Білик, В.Б. Матикін

У роботі проведено виділення рівнів ієрархій інформаційних зв'язків, окремих задач комплексної оиінки осередків ядерного ураження та пов'язуючих їх інформачійних потоків. Аналізуються причинно-наслідкові зв 'язки множини основних параметрів, характер залежності між рівнем цінності необхідної інформації та ї̈ кількістю в системі радіаційного моніторингу. Апробована методологія кількісної оцінки повного і точного обліку необхідних параметрів у завданні комплексної оиінки осередків ядерного ураження. Авторами пропонується методологія комплексної оцінки осередків ядерного ураження на основі реєстрачії та обліку необхідних і достатніх параметрів ядерного вибуху, параметрів середовища і параметрів об'єкта у зоні надзвичайної ситуачії.

Ключові слова: теоретичні і методологічні основи побудови, надзвичайна ситуачія воєнного характеру, радіаційний моніторинг, системно-інформачійний аналіз, осередкі ядерного ураження, параметри ядерного вибуху, виявлення $i$ оцінка обстановки.

\section{USE OF RESULTS IN THE DEVELOPMENT OF THEORETICAL AND METHODOLOGICAL BASES OF CONSTRUCTION RADIATION MONITORING SYSTEM OF EMERGENCIES WHICH HAS A MILITARY NATURE}

I.Yu. Cherniavskiy, V.V. Tyutyunik, V.D. Kalugin, Z.V. Bilyk, V.B. Matykin

The work carried out the selection of levels of hierarchy of information links, individual problems of the integrated assessment of nuclear foci and the information flows linking them. The cause-effect relationships of many basic parameters, the nature of the relation between the level of value of the necessary information and its quantity in the radiation monitoring system are analyzed. The methodology of a quantitative assessment of the complete and accurate accounting of the necessary parameters in the problem of a complex assessment of focus of nuclear damage is approved.The authors propose a methodology for a integrated assessment of nuclear foci on the basis of accounting and recording the necessary and sufficient parameters of a nuclear explosion, the parameters of the environment and the parameters of the object in the emergency zone.

Keywords: theoretical and methodological bases of construction, emergency situations of a military nature, radiation monitoring, system and information analysis, the focus of nuclear damage, parameters of a nuclear explosion, identification and assessment of the situation. 